\title{
Pesticide contamination and associated risk factors at public playgrounds near intensively managed apple and wine orchards
}

\author{
Caroline Linhart ${ }^{1 *}$, Georg H. Niedrist ${ }^{2}$, Magdalena Nagler ${ }^{3}$, Rajini Nagrani ${ }^{4,5}$, Veronika Temml ${ }^{1}$, \\ Tommaso Bardelli ${ }^{3,6}$, Thomas Wilhalm7, Andreas Riedl ${ }^{7}$, Johann G. Zaller ${ }^{8}$, Peter Clausing ${ }^{9}$ and Koen Hertoge ${ }^{10^{*}}$
}

\begin{abstract}
Background: Pesticide levels are generally monitored within agricultural areas, but are commonly not assessed at public places. To assess possible contamination of non-target areas, 71 public playgrounds located next to intensively managed apple and wine orchards were selected in four valleys of South Tyrol (northern Italy). Further, the impact of environmental site characteristics on the number and concentration of pesticides was assessed. Grass samples from the selected playgrounds were collected and screened for 315 pesticide residues using standard gas chromatography and mass spectrometry.

Results: Nearly half of the playgrounds (45\%) were contaminated by at least one pesticide and a quarter (24\%) by more than one. Eleven of the 12 different detected pesticides are classified as endocrine-active substances including the insecticide phosmet and the fungicide fluazinam showing the highest concentrations ( 0.069 and $0.26 \mathrm{mg} \mathrm{kg}^{-1}$, respectively). Additionally, one disinfectant and one preservation agent was found. Playgrounds in Venosta valley were most often contaminated (76\% of all investigated playgrounds), highest concentrations were found in the Low Adige $\left(2.02 \mathrm{mg} \mathrm{kg}^{-1}\right)$. Pesticide concentrations were positively associated with areal proportion of apple orchards in the surroundings, the amount of rainfall and wind speed. In contrast, increasing global irradiance, opposite wind direction, increasing distance to agricultural sites and high wind speeds when pesticide application was not allowed were associated with decreasing pesticide contamination.
\end{abstract}

Conclusion: This study is among the first investigating pesticide contamination of public playgrounds together with environmental factors in areas with pesticide-intensive agriculture at the beginning of the growing season. It is likely that playgrounds will be affected by more pesticides and higher concentrations over the course of the crop season. The result, that the majority of the detected pesticides are classified as endocrine active is worrisome as children are especially vulnerable. Hence, we recommend that pesticide risk assessments should better include protection measures for non-target areas.

Keywords: Non-targeted area contamination, Fungicides, Insecticides, Fluazinam, Phosmet, Endocrine disruptors, Pesticide drift

\footnotetext{
${ }^{*}$ Correspondence: caroline.linhart@uibk.ac.at; koen.hertoge@gmail.com

${ }^{1}$ Institute of Pharmacy/Pharmacognosy, Leopold-Franzens University

Innsbruck, Innsbruck, Austria

${ }^{10}$ Pesticide Action Network Europe, Brussels, Belgium

Full list of author information is available at the end of the article
}

\section{Springer Open}

C The Author(s) 2019. This article is distributed under the terms of the Creative Commons Attribution 4.0 International License (http://creativecommons.org/licenses/by/4.0/), which permits unrestricted use, distribution, and reproduction in any medium, provided you give appropriate credit to the original author(s) and the source, provide a link to the Creative Commons license, and indicate if changes were made. 


\section{Background}

Pesticides are globally used substances aimed to fight various pests and diseases [13]. Chemically, the principal classes of currently used synthetic pesticides are, beside others, organochlorines, organophosphates, carbamates, neonicotinoids and pyrethroids [34]. In 2012, about $2600 \times 10^{6} \mathrm{~kg}$ of pesticides were used worldwide [5] and in $2014,396 \times 10^{6} \mathrm{~kg}$ of pesticides were applied in the European Union [31, 61]. Besides their designated function, pesticides and their residues remain active and can drift far beyond their target areas via air, water, soil erosion or leaching [58].

Studies have shown that pesticides affect non-target organisms including crop species [84], microorganisms and invertebrates $[43,73,85]$, with negative consequences for the overall biodiversity in various ecosystems $[41,70]$.

Pesticides are associated with both, acute and delayed health effects in exposed humans, ranging from simple skin and eye irritation to more severe impacts on the nervous and the reproductive system [22, 74]. Especially occupational exposure to pesticides is associated with the development of a wide spectrum of pathologies [34], such as Parkinson's disease among winemakers [44], myocardial infarction among female farmers [80] and cardiovascular disease among farm workers [68]. In addition, occupational pesticide exposure was found to increase the general risk of cancer [2, 45]. More than 100 pesticides are considered as endocrine active that interfere with oestrogen or androgen receptors [54] linking pesticides classified as endocrine disruptors (EDs) to an increased risk for breast cancer [24]. Next to the occupational pesticide exposure, indirect exposure via air drift, water and nutrition may affect humans living near intensively managed agricultural areas. For example, long-term, non-occupational exposure to pesticides contributes to the development of chronic diseases [14, $56,82]$ or of non-communicable diseases such as obesity, diabetes, thyroid diseases, cancers and especially hormonal-related cancers $[8,17,20,55]$. Exposure to a variety of different pesticides might also trigger synergistic effects [48], which are extremely difficult to anticipate [18].

The exposure of children to EDs is of particular concern, as they are exposed during sensitive development stages, increasing the risk of adverse health effects compared to adults [66]. For children, the risk for cancer is even associated with parental exposure to occupational or non-occupational pesticide [7, 74]. Evaluations and measurements of potential hazards for children are essential to conduct health risk assessments of pesticides. For that it is crucial to consider the often neglected, low-dose and diffuse pesticide exposure via spray drift. While this inconspicuous exposure might not yield great amounts of pesticides, the cumulated effect of multiple pesticides might pose risks for human health [10].Children are often in direct contact with soil, plants or toy surfaces, especially while playing on playgrounds [66, 81].

In the light of health concerns regarding indirect pesticide exposure and risk for children, the present study aims to assess exposure levels of public children playgrounds in an intensively managed apple and winegrowing region in northern Italy, one of the leading apple- and wine-producing areas in Europe [30]. While Italy shows higher pesticide usage compared to other European countries [31], the Trentino-Alto Adige Region and the autonomous province of Bolzano-Bozen show considerably high usage, reporting an average usage of $21.6 \mathrm{~kg} \mathrm{ha}^{-1}$ fungicides, $13.1 \mathrm{~kg} \mathrm{ha}^{-1}$ insecticides and $0.9 \mathrm{~kg} \mathrm{ha}^{-1}$ herbicides in 2017 [42]. Due to limited cultivable and habitable land in the mountainous province of Bolzano-Bozen there is often inevitable spatial proximity of agricultural areas and human settlements.

To assess the susceptibility of public non-agricultural areas for pesticide drift and to examine potential underlying factors, we investigated playgrounds around apple and vine orchards as well as their local and meteorological conditions. We hypothesise that (i) a higher pesticide exposure will occur at public playgrounds closer to intensively managed apple and vine orchards and that (ii) location and local agricultural management as well as (iii) environmental and meteorological characteristics including irradiance, precipitation level, wind speed/direction, and air temperature will affect potential pesticide exposure. To test these hypotheses and to examine how a possible pesticide contamination could be minimised, we investigated four intensively used agricultural areas in the province of South Tyrol (northern Italy) and their specific environmental factors.

\section{Materials and methods Site description}

We identified 125 potential playgrounds in the valleys Venosta (Vinschgau), Isarco (Eisack), Adige (Etsch), and Low Adige (Südtiroler Unterland) using official web pages listing public playgrounds $[60,78]$ and 71 of these playgrounds were randomly selected for sampling (Fig. 1). Randomisation and sampling site selection were done separately for each geographical area, considering an equal distribution of the mean distance to agricultural fields in the four sampling regions (Additional file 2: Fig S2).

Generally, the four investigated valleys differ in land use, mean air temperature, mean precipitation and altitude (Additional file 1: Fig S1, Additional file 2: Fig S2, Additional file 3: Fig S3). The Adige valley and the Low Adige have mean annual temperatures of $12{ }^{\circ} \mathrm{C}$ 


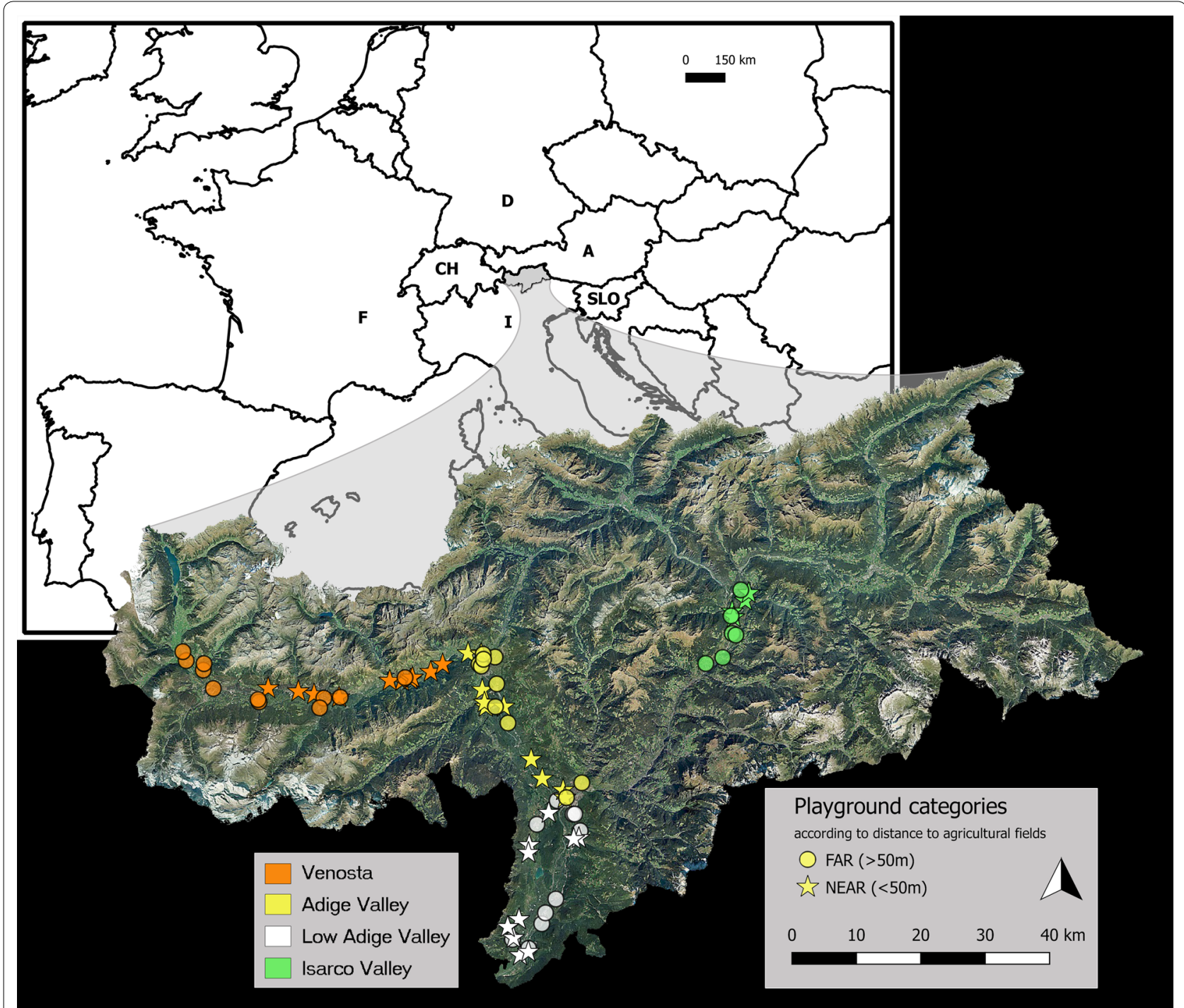

Fig. 1 Map of the province Bolzano-Bozen in the Italian region Trentino-South Tyrol. Sampled playgrounds are distributed in four valleys of the province and were classified as FAR and NEAR sites (with less and more than $50 \mathrm{~m}$ distance from agricultural fields, respectively). Orange $=$ Venosta valley, yellow $=$ Adige valley, white $=$ Low Adige, green $=$ Isarco valley

and mean annual precipitation of $720 \mathrm{~mm}$ and $856 \mathrm{~mm}$, respectively. Sampled playgrounds in the Adige valley were located at a mean altitude of $287 \mathrm{~m}$ above sea level (a.s.l.; min 250, max 350) and in the Low Adige at $293 \mathrm{~m}$ a.s.l. (min 200, $\max 610$, Additional file 1: Fig. S1). The Low Adige is dominated by vine yards (20\%), while the agriculture of the Adige valley is dominated by apple orchards (32\%, Additional file 3: Fig. S3). In comparison, the Venosta and the Isarco valley exhibit lower mean annual temperatures $\left(8.8^{\circ} \mathrm{C}\right.$ and $10.1^{\circ} \mathrm{C}$, respectively) and annual precipitation (566 and $670 \mathrm{~mm}$, respectively), while the sampled playgrounds in these valleys were located at higher altitudes, with
$688 \mathrm{~m}$ a.s.l. (min 555, $\max 890)$ and $737 \mathrm{~m}$ a.s.l. (min 515, max 1130, Additional file 1: Fig S1), respectively. Apple cultivation is the dominant land use type in the Venosta and the Isarco valley $(20-30 \%$ of the total land cover), while cultivation of wine has a less important role (Additional file 3: Fig. S3).

Geographical data including elevation, distance to nearest orchards and direction of closest or chards were obtained from the regional geographic information system Geobrowser [6]. Meteorological data including temperature, global irradiance, wind speed, wind direction and precipitation were obtained from the nearest meteorological stations calculated for 14 
and 5 days prior to sampling provided by the regional hydrographic service [36].

Pesticide spraying is recommended during wind speeds $<3 \mathrm{~m} \mathrm{~s}^{-1}[1,21]$. Therefore, data on wind was additionally separated in hours while spraying was recommended (i.e. $<3 \mathrm{~m} \mathrm{~s}^{-1}$ ) and hours while spraying was not recommended (i.e. $\geq 3 \mathrm{~m} \mathrm{~s}^{-1}$ ).

Land use data within a $500 \mathrm{~m}$ radius around sampled sites was provided by the Federal Agricultural Office of the Autonomous Province of Bolzano-South Tyrol. This data included detailed information on the area of apple and wine or chards other fruit orchards, other agricultural areas (e.g., arable crops, grassland), forests, buildings and infrastructure, fallow land and waterbodies $\left(\mathrm{m}^{2}\right)$.

According to the distance to nearest agricultural fields, sampled playgrounds were classified as NEAR $(\leq 50 \mathrm{~m}$ distance) and FAR ( $>50 \mathrm{~m}$ distance). The distance was measured from the centre of the playground to the first apple tree or grape vine of the adjacent agricultural area. The agricultural areas considered in this study included both, conventionally and organically farmed apple orchards and vineyards. The final randomized selection included 21 sampling sites in the Venosta (10 NEAR, 11 FAR), 20 in the Adige valley (10 NEAR, 10 FAR), 20 in Low Adige (10 NEAR, 10 FAR) and 10 in the Isarco valley (4 NEAR, 6 FAR), resulting in a total of 34 NEAR and 37 FAR sites.

\section{Sampling and analysis}

Plant sampling was conducted between May 16-23, 2017, on dry days after a period of at least five rainless days, by the accredited office BioProgramm from Padova [11]. For this purpose, 3 to 4 randomly selected grass samples per playground were taken by hand-wearing laboratory gloves and grouped to one 300-400 g (fresh mass) composite sample. Plant material was vacuumpacked in freezer bags and stored at room temperature until analysis by the laboratory for food analysis of the Autonomous Province of Bolzano. After acetonitrile extraction (701881-Acetonitrile for LC-MS, ITW Reagents PanReac AppliChem) according to the dispersive SPE-QuEChERS-method [3], pesticide concentrations of grass samples were analysed using Agilent, $7000 \mathrm{C}$ Triple Quadrupole GC/MS series system, (Agilent, Waldbronn, Germany) and API $4000^{\mathrm{TM}} \mathrm{LC}-\mathrm{MC} / \mathrm{MS}$ series system (AB Sciex, Concord ON, Canada), following the European standard UNI EN 15662:2009 [64] to identify up to 315 substances. Measured pesticide concentrations are based on wet weight of fresh grass samples.

\section{Statistical analyses}

We conducted the statistical analysis according to a predefined statistical analysis plan to identify environmental mediators of pesticide concentrations and to predict the environmental conditions associated with the lowest levels of pesticide pollution. Continuous variables were presented as medians with interquartile range (IQR) and categorical variables as percentages. Descriptive statistics, analyses of variance (ANOVA) and regression analysis were performed using R v. 3.5.1 [65], considering normal distribution of data or homogeneity of variances. Non-normal distributed factors, irradiance and wind, were log-transformed before analysis.

Chi squared test and Fisher's exact test for count data was applied for the categorical data number of detected pesticides and independent 2-group Mann-Whitney $U$ Test for zero-inflated continuous data on pesticide concentrations between NEAR and FAR sites. Kruskal-Wallis Test, one way ANOVA by ranks and, if main effects were found, a post hoc test (Tukey and Dunn's test) was used to compare pesticide contamination among the four different regions. An initial univariate hurdle model, with distance as predictor, was used to (i) analyse the association of spatial proximity and pesticide concentration levels (linear model using non-zero values) and (ii) to assess how distance is associated with the probability of playgrounds to be contaminated (binomial model).

Based on this, a multivariate weighted linear regression analysis was performed, to estimate the effect of environmental factors on the measured pesticide concentrations $\left(\mathrm{mg} \mathrm{kg}{ }^{-1}\right)$ weighted for number of detected pesticides. The model containing first-order interactions evaluated the impact of the following environmental factors: precipitation $(\mathrm{mm})$, air temperature $\left({ }^{\circ} \mathrm{C}\right)$, global irradiance $\left(\mathrm{W} \mathrm{m}^{-2}\right.$ ), altitude ( $\mathrm{m}$ a.s.l.), wind direction, wind speed $\left(\mathrm{m} \mathrm{s}^{-1}\right)$, orientation of the playground to the surrounding agricultural sites (16 cardinal points), hours of possible drift $(\mathrm{h})$ and the relative area covered by forest, apple and wine orchards within a radius of $500 \mathrm{~m}(\%)$.

Additionally, the sum of windy hours and averaged maximum wind speed per wind direction (from or to the agricultural field) were calculated for the 5- and 14-day period before sampling. Further, hours with wind speeds higher and lower than $3 \mathrm{~m} \mathrm{~s}^{-1}$ were summed separately as spraying is not allowed with wind speeds $>3 \mathrm{~m} \mathrm{~s}^{-1}$.

The weighting of the dependent variable pesticide concentration was based on the number of detected pesticide species per playground. Thus, the dependent variable-the total measured pesticide concentration-was weighted for the detected number of different pesticides per playground. Stepwise variable selection was conducted using the Akaike information criterion (AIC) and the Bayesian information criterion (BIC) for model building, considering multi-collinearity and variance inflation factors (VIFs; only variables with a VIF $<3$ were included in the final model; 
[88]). For the analysis, all descriptors were centred and scaled for model analytics, but were retransformed for result presentation via effect- and interaction plots.

Model evaluation and control for over-dispersion was done by analysis of deviance with the anova() function [88]. Homoscedasticity of the models was checked by Breusch-Pagan test. Model evaluation and final selection was based on comparisons of $R^{2}$, adjusted $R^{2}, A I C$ and BIC. Correction method for multiple testing (post hoc tests and Welch correction) was applied where necessary. Two-sided $p$ values $<0.05$ were considered statistically significant.

\section{Results}

Pesticides were detected in $32(45 \%)$ of 71 playgrounds, of which 17 (24\% of all sites) contained more than one type of pesticides, seven contained three types, and two sites contained four pesticides (Table 1). In sum, 12 different pesticides were identified within the study sites, including five insecticides (phosmet, imidacloprid, chlorpyrifos-methyl, methoxyfenozid and cypermethrin), six fungicides (fluazinam, dodine, difenoconazole, penconazole, tetraconazole and penthiopyrad), and one herbicide (oxadiazon). Except dodine, all other 11 of the 12 pesticides are associated with endocrine activity. Additionally, one preservative agent (2-phenylphenol) and one disinfectant (benzalkonium chloride) was found.

The insecticide phosmet and the fungicide fluazinam were the most abundant pesticides, with median concentrations of $0.013 \mathrm{mg} \mathrm{kg}^{-1}$ and $0.012 \mathrm{mg} \mathrm{kg}^{-1}$ and maximum concentrations of 0.069 and $0.26 \mathrm{mg} \mathrm{kg}$, respectively (Table 2).

Table 1 Descriptive statistics of pesticides detected in grass samples of playgrounds $(n=71)$

\begin{tabular}{|c|c|c|c|c|}
\hline Pesticides and pesticide classes & $\begin{array}{l}\text { Total }(N=71) \\
n(\%)\end{array}$ & $\begin{array}{l}\text { NEAR }(N=34) \\
n(\%)\end{array}$ & $\begin{array}{l}\text { FAR }(N=37) \\
n(\%)\end{array}$ & $p$ value \\
\hline \multicolumn{5}{|l|}{ Number of contaminated playgrounds* } \\
\hline Present & $32(45.1)$ & $21(61.8)$ & $11(29.7)$ & 0.013 \\
\hline \multicolumn{5}{|l|}{ Playgrounds with } \\
\hline No pesticides & $39(54.9)$ & $13(38.2)$ & $26(70.3)$ & 0.043 \\
\hline One pesticide & $15(21.1)$ & $8(23.5)$ & $7(18.9)$ & \\
\hline Two pesticides & $8(11.3)$ & $6(17.7)$ & $2(5.4)$ & \\
\hline Three pesticides & $7(9.9)$ & $5(14.7)$ & $2(5.4)$ & \\
\hline Four pesticides & $2(2.8)$ & $2(5.9)$ & 0 & \\
\hline \multicolumn{5}{|l|}{ Detected pesticides } \\
\hline \multicolumn{5}{|l|}{ Herbicides } \\
\hline Oxadiazon & $1(1.4)$ & $1(2.9)$ & 0 & 0.479 \\
\hline Insecticides* & $22(31.0)$ & $14(41.2)$ & $8(21.6)$ & 0.128 \\
\hline Phosmet* & $18(25.4)$ & $11(32.4)$ & $7(18.9)$ & 0.305 \\
\hline Imidacloprid & $3(4.2)$ & $2(5.9)$ & $1(2.7)$ & 0.604 \\
\hline Chlorpyrifos-methyl & $1(1.4)$ & $1(2.9)$ & 0 & 0.479 \\
\hline Methoxyfenozide & $1(1.4)$ & $1(2.9)$ & 0 & 0.479 \\
\hline Cypermethrin & $1(1.4)$ & $1(2.9)$ & 0 & 0.479 \\
\hline Fungicides* & $21(29.6)$ & $17(50)$ & $4(10.8)$ & $<0.001$ \\
\hline Fluazinam* & $18(25.4)$ & $14(41.2)$ & $4(10.8)$ & 0.008 \\
\hline Penthiopyrad & $6(8.5)$ & $6(17.7)$ & 0 & 0.009 \\
\hline Difenoconazole & $2(2.8)$ & $2(5.9)$ & 0 & 0.223 \\
\hline Dodine & $1(1.4)$ & $1(2.9)$ & 0 & 0.479 \\
\hline Penconazole & $2(2.8)$ & $1(2.9)$ & $1(2.7)$ & 0.999 \\
\hline Tetraconazole & $1(1.4)$ & $1(2.9)$ & 0 & 0.479 \\
\hline \multicolumn{5}{|l|}{ Disinfectant } \\
\hline Benzalkonium chloride & $4(5.6)$ & $1(2.9)$ & $3(8.1)$ & 0.616 \\
\hline \multicolumn{5}{|l|}{ Preservative agent } \\
\hline 2-Phenylphenol & $1(1.4)$ & 0 & $1(2.7)$ & 0.999 \\
\hline Presence of pesticides with endocrine activity* & $26(36.6)$ & $17(50)$ & $9(24.3)$ & 0.046 \\
\hline
\end{tabular}

Grass samples of playgrounds were stratified by distance from agricultural fields: $\leq 50 \mathrm{~m}=\mathrm{NEAR},>50 \mathrm{~m}=$ FAR. All $p$-values are by Fisher's-exact test unless indicated with an *asterisk (Chi squared test) 


\section{Sites NEAR vs. FAR from agricultural sites}

Median and inter-quartile ranges of pesticide concentrations in NEAR sites $\left(0.04 \mathrm{mg} \mathrm{kg}^{-1}, 0.03-0.08\right)$ were significantly higher than in FAR sites $\left(0.01 \mathrm{mg} \mathrm{kg}^{-1}, 0.01-\right.$ 0.04, Mann-Whitney $U=58, n_{1}=21, n_{2}=11, p=0.024$, $r=0.4$, Table 2). Similarly, the number of sites containing pesticides was significantly higher in NEAR (61.8\%) compared to FAR sites $\left(29.7 \%, p_{X}^{2}=0.013\right)$ (Table 1$)$.

More specifically, NEAR sites showed significantly more pesticides and higher concentrations of fluazinam $\left(p_{X}^{2}=0.008\right.$ and $P_{\text {MannWhitney } U}=0.003$, resp. $)$ and penthiopyrad $\left(P_{\text {FishersExact }}=0.009, P_{\text {MannWhitney } U}=0.008\right)$, than FAR sites (Tables 1 and 2).

Endocrine-active pesticides were found more often and in higher concentrations in NEAR (50\% and median $0.03 \mathrm{mg} \mathrm{kg} \mathrm{kg}^{-1}$ ) as compared to FAR sites (24\%, and median $0.01 \mathrm{mg} \mathrm{kg}-1, P_{\text {FishersExact }}=0.029$, Table 1 and $P_{\text {MannWhitney } U}=0.006$, Table 2).

\section{Pesticide records in the four study regions}

The playgrounds of the four selected study areas differed significantly in their pesticide contaminations: while in the Venosta valley $76 \%$ of playgrounds showed pesticides (Fisher's exact test, $p=0.003$ ), only $40 \%$ of playground in Adige valley and Isarco valley had a pesticide contamination; in the Low Adige only 20\% of the inspected playgrounds showed pesticide residues (Table 3). Additionally, the concentration levels of detected pesticides differed significantly between the four selected study sites ( $P_{\text {KruskalWallis }}=0.025$, Table 4$)$, recording the highest concentration level in the Low Adige with a median of $0.19 \mathrm{mg} \mathrm{kg}^{-1}$, although having the least number of contaminated sites.

Sampled playgrounds are stratified according to the four investigated valleys. Fisher's-exact test and post hoc pairwise test for nominal data (adj. Fisher's Exact for multiple comparisons).

The insecticide phosmet was detected on 13 playgrounds in Venosta, being significantly more frequent $\left(P_{\text {FishersExact }}<0.001\right.$, Table 3$)$ and higher concentrated $\left(P_{\text {KruskalWallis }}<0.001\right.$, Table 4$)$ as compared to other regions.

The fungicide fluazinam was significantly more frequent (10 detections) in the Venosta valley, but with $0.013 \mathrm{mg} \mathrm{kg}^{-1}$ not significantly higher concentrated than in the other regions $\left(P_{\text {FishersExact }}=0.048\right.$, Table 3 and $P_{\text {KruskalWallis }}=0.903$, Table 4 , respectively).

Penthiopyrad, however, was detected more often in the Isarco valley (4 detections, $P_{\text {FishersExact }}=0.004$, Table 3) with significantly higher concentrations (0.036 $\mathrm{mg} \mathrm{kg}^{-1}, P_{\text {Kruskalwallis }}=0.001$, Post Hoc test: $p<0.05$ ) than in other regions. (Table 3 ).

Difenoconazol was only detected in the Isarco valley at two playgrounds and benzalkoniumchloride was only detected in the Adige valley.

\section{Pesticide contamination and local conditions}

Pesticide concentration, weighted for the number of pesticides detected per playground, was strongly dependent on the following local environmental parameters

Table 2 Medians, interquartile ranges (in parentheses) and maximum concentrations (italics) of pesticide concentrations ( $\mathrm{mg} \mathrm{kg}^{-1}$ wet weight)

\begin{tabular}{|c|c|c|c|c|c|}
\hline Pesticides and pesticide classes & Total $(N=32)$ & $\operatorname{NEAR}(N=21)$ & $\operatorname{FAR}(N=11)$ & $p$-value $(N=32)^{\mathrm{a}}$ & $p$-value $(n=71)^{\mathrm{b}}$ \\
\hline Pesticide concentration & $\begin{array}{l}0.035(0.016-0.067) \\
2.024\end{array}$ & $\begin{array}{l}0.041(0.029-0.082) \\
2.024\end{array}$ & $\begin{array}{l}0.014(0.013-0.039) \\
0.117\end{array}$ & 0.024 & 0.001 \\
\hline Insecticide concentration & $\begin{array}{l}0.014(0.00-0.027) \\
1.9\end{array}$ & $\begin{array}{l}0.018(0.00-0.032) \\
1.9\end{array}$ & $\begin{array}{l}0.014(0.006-0.017) \\
0.05\end{array}$ & 0.431 & 0.036 \\
\hline Fungicide concentration & $\begin{array}{l}0.013(0.00-0.036) \\
0.325\end{array}$ & $\begin{array}{l}0.02(0.011-0.048) \\
0.325\end{array}$ & $\begin{array}{l}0.00(0.00-0.012) \\
0.063\end{array}$ & 0.010 & $<0.001$ \\
\hline Pesticides with endocrine activity & $\begin{array}{l}0.032(0.014-0.067) \\
1.93\end{array}$ & $\begin{array}{l}0.033(0.018-0.068) \\
1.93\end{array}$ & $\begin{array}{l}0.014(0.011-0.023) \\
0.117\end{array}$ & 0.071 & 0.006 \\
\hline \multicolumn{6}{|c|}{ Concentrations of most abundant pesticides } \\
\hline Phosmet & $\begin{array}{l}0.013(0.00-0.019) \\
0.069\end{array}$ & $\begin{array}{l}0.011(0.00-0.019) \\
0.069\end{array}$ & $\begin{array}{l}0.013(0.00-0.017) \\
0.054\end{array}$ & 0.983 & 0.148 \\
\hline Fluazinam & $\begin{array}{l}0.012(0.00-0.019) \\
0.26\end{array}$ & $\begin{array}{l}0.014(0.00-0.022) \\
0.26\end{array}$ & $\begin{array}{l}0.00(0.00-0.007) \\
0.049\end{array}$ & 0.068 & 0.003 \\
\hline Penthiopyrad & $\begin{array}{l}0.0(0.00-0.00) \\
0.1\end{array}$ & $\begin{array}{l}0.0(0.00-0.011) \\
0.1\end{array}$ & 0 & 0.058 & 0.008 \\
\hline
\end{tabular}

Contaminated playgrounds $(N=32)$ were stratified by distance from agricultural fields: $\leq 50 \mathrm{~m}=\mathrm{NEAR},>50 \mathrm{~m}=\mathrm{FAR}$

a Mann-Whitney $U$ test with continuity correction

b Including all sampling sites $(n=71)$ 
Table 3 Number and percentage (\%) of pesticides detected in grass samples from playgrounds $(n=71)$

\begin{tabular}{|c|c|c|c|c|c|c|}
\hline $\begin{array}{l}\text { Pesticides/pesticide } \\
\text { classes }\end{array}$ & & $\begin{array}{l}\text { Isarco valley } \\
(N=10)\end{array}$ & $\begin{array}{l}\text { Adige valley } \\
(N=20)\end{array}$ & $\begin{array}{l}\text { Low Adige } \\
(N=20)\end{array}$ & $\begin{array}{l}\text { Venosta } \\
(N=21)\end{array}$ & $p$-value \\
\hline \multirow{3}{*}{$\begin{array}{l}\text { Contaminated play- } \\
\text { grounds }\end{array}$} & & $4(40.00)$ & $8(40.00)$ & $4(20.00)$ & $16(76.19)$ & 0.003 \\
\hline & & Post hoc test & Low Adige: Ve & & & 0.003 \\
\hline & & & Other compar & & & n.s. \\
\hline \multicolumn{7}{|l|}{ Playgrounds with } \\
\hline & No pesticides & $6(60)$ & $12(60)$ & $16(80)$ & $5(23.8)$ & \multirow[t]{5}{*}{0.005} \\
\hline & One pesticide & $2(20)$ & $5(25)$ & 0 & $8(38.1)$ & \\
\hline & Two pesticides & 0 & $2(10)$ & $1(5)$ & $5(23.8)$ & \\
\hline & Three pesticides & $1(10)$ & $1(5)$ & $3(15)$ & $2(9.5)$ & \\
\hline & Four pesticides & $1(10)$ & 0 & 0 & $1(4.8)$ & \\
\hline & & \multirow[t]{2}{*}{ Post hoc test } & \multicolumn{3}{|c|}{ Low Adige: Venosta } & $<0.001$ \\
\hline & & & \multicolumn{3}{|c|}{ Other comparisons } & n.s. \\
\hline \multicolumn{7}{|l|}{ Pesticide classes } \\
\hline Herbicides & Oxadiazon & 0 & 0 & 0 & $1(4.76)$ & 0.999 \\
\hline \multirow[t]{12}{*}{ Insecticides } & & $1(10)$ & $4(20)$ & $4(20)$ & $13(61.9)$ & 0.004 \\
\hline & & Post hoc test & \multicolumn{3}{|c|}{ Venosta: all other regions } & 0.022 \\
\hline & & & \multicolumn{3}{|c|}{ Other comparisons } & n.s. \\
\hline & Phosmet & 0 & $4(20.00)$ & $1(5.00)$ & $13(61.90)$ & $<0.001$ \\
\hline & & Post hoc test & \multicolumn{3}{|c|}{ Isarco valley: Venosta } & 0.004 \\
\hline & & & \multicolumn{3}{|l|}{ Adige: Venosta } & 0.002 \\
\hline & & & \multicolumn{3}{|c|}{ Low Adige: Venosta } & $<0.001$ \\
\hline & & & \multicolumn{3}{|c|}{ Other comparisons } & n.s. \\
\hline & Imidacloprid & $1(10.00)$ & 0 & $2(10.00)$ & 0 & 0.719 \\
\hline & Chlorpyrifos-methyl & 0 & 0 & $1(5.00)$ & 0 & 0.704 \\
\hline & Methoxyfenozide & 0 & 0 & $1(5.00)$ & 0 & 0.704 \\
\hline & Cypermethrin & 0 & 0 & $1(5.00)$ & 0 & 0.704 \\
\hline \multirow[t]{12}{*}{ Fungicides } & & $4(40)$ & $4(20)$ & $2(10)$ & $11(52.4)$ & 0.015 \\
\hline & & Post hoc test & \multicolumn{3}{|c|}{ Venosta: all other regions } & $<0.001$ \\
\hline & & & \multicolumn{3}{|c|}{ Other comparisons } & n.s. \\
\hline & Fluazinam & $2(20.00)$ & $4(20.00)$ & $2(10.00)$ & $10(47.62)$ & 0.048 \\
\hline & & Post hoc test & \multicolumn{3}{|c|}{ Low Adige: Venosta } & 0.015 \\
\hline & & & \multicolumn{3}{|c|}{ Other comparisons } & n.s. \\
\hline & Penthiopyrad & $4(40.00)$ & 0 & $1(5.00)$ & $1(4.76)$ & 0.004 \\
\hline & & Post hoc test & \multicolumn{3}{|c|}{ Isarco valley: Adige valley } & 0.046 \\
\hline & Difenoconazole & $2(20.00)$ & 0 & 0 & 0 & 0.018 \\
\hline & Dodine & 0 & 0 & $1(5.00)$ & 0 & 0.704 \\
\hline & Penconazole & 0 & 0 & 0 & $2(9.52)$ & 0.501 \\
\hline & Tetraconazole & 0 & 0 & 0 & $1(4.76)$ & 0.999 \\
\hline \multicolumn{7}{|l|}{ Disinfectant } \\
\hline & $\begin{array}{l}\text { Benzalkonium } \\
\text { chloride }\end{array}$ & 0 & $4(20.00)$ & 0 & 0 & 0.018 \\
\hline \multicolumn{7}{|l|}{ Preservative agent } \\
\hline & 2-Phenylphenol & 0 & 0 & $1(5.00)$ & 0 & 0.704 \\
\hline Pesticides with EA & & $2(20)$ & $5(25)$ & $4(20)$ & $15(71.4)$ & 0.002 \\
\hline & & Post hoc test & Isarco: Venosta & & & 0.036 \\
\hline & & & Adige: Venost & & & 0.014 \\
\hline & & & Low Adige: Ve & & & 0.009 \\
\hline & & & Other compar & & & n.s. \\
\hline
\end{tabular}


Table 4 Medians, interquartile ranges (in parentheses) and maxima (italics) of pesticide concentrations ( $\mathrm{mg} \mathrm{kg}^{-1}$ wet weight)

\begin{tabular}{|c|c|c|c|c|c|c|}
\hline $\begin{array}{l}\text { Pesticides } \\
\text { and pesticide } \\
\text { classes }\end{array}$ & Isarco valley $(n=4)$ & Adige valley $(n=8)$ & Low Adige $(n=4)$ & Venosta $(n=16)$ & $p$-value $(n=32)^{\mathrm{a}}$ & $p$-value $(N=71)^{b}$ \\
\hline \multirow[t]{2}{*}{$\begin{array}{l}\text { Pesticide concentra- } \\
\text { tion }\end{array}$} & $\begin{array}{l}0.084(0.061-0.116) \\
0.163\end{array}$ & $\begin{array}{l}0.024(0.012-0.032) \\
0.048\end{array}$ & $\begin{array}{l}0.186(0.061-0.734) \\
2.024\end{array}$ & $\begin{array}{l}0.032(0.014-0.053) \\
0.375\end{array}$ & 0.014 & 0.025 \\
\hline & Post hoc test & \multicolumn{3}{|l|}{ Adige valley: Low Adige } & 0.047 & \\
\hline \multirow[t]{2}{*}{$\begin{array}{l}\text { Insecticide concen- } \\
\text { tration }\end{array}$} & $\begin{array}{l}0(0-0.006) \\
0.023\end{array}$ & $\begin{array}{l}0.006(0-0.018) \\
0.019\end{array}$ & $\begin{array}{l}0.159(0.055-0.663) \\
1.9\end{array}$ & $\begin{array}{l}0.016(0.013-0.03) \\
0.069\end{array}$ & 0.026 & 0.008 \\
\hline & Post hoc test & \multicolumn{3}{|c|}{ Adige valley: Low Adige } & 0.049 & \\
\hline $\begin{array}{l}\text { Fungicide concentra- } \\
\text { tion }\end{array}$ & $\begin{array}{l}0.72(0.041-0.116) \\
0.163\end{array}$ & $\begin{array}{l}0.006(0-0.148) \\
0.028\end{array}$ & $\begin{array}{l}0.027(0-0.072) \\
0.124\end{array}$ & $\begin{array}{l}0.013(0-0.02) \\
0.325\end{array}$ & 0.061 & 0.027 \\
\hline $\begin{array}{l}\text { Pesticides with endo- } \\
\text { crine activity }\end{array}$ & $\begin{array}{l}0.025(0.00-0.135) \\
0.135\end{array}$ & $\begin{array}{l}0.02(0.0-0.03) \\
0.039\end{array}$ & $\begin{array}{l}0.168(0.054-0.684) \\
1.933\end{array}$ & $\begin{array}{l}0.032(0.014-0.053) \\
0.375\end{array}$ & 0.096 & 0.005 \\
\hline \multicolumn{7}{|c|}{ Concentrations of most abundant pesticides } \\
\hline \multirow[t]{2}{*}{ Phosmet } & $\begin{array}{l}0(0-0) \\
0\end{array}$ & $\begin{array}{l}0.006(0-0.018) \\
0.019\end{array}$ & $\begin{array}{l}0(0-0.009) \\
0.036\end{array}$ & $\begin{array}{l}0.015(0.013-0.03) \\
0.069\end{array}$ & 0.043 & $<0.001$ \\
\hline & Post hoc test & \multicolumn{2}{|l|}{ Isarco valley: Venosta } & 0.014 & & \\
\hline Fluazinam & $\begin{array}{l}0.007(0-0.041) \\
0.12\end{array}$ & $\begin{array}{l}0.006(0-0.015) \\
0.028\end{array}$ & $\begin{array}{l}0.009(0-0.022) \\
0.033\end{array}$ & $\begin{array}{l}0.013(0-0.02) \\
0.26\end{array}$ & 0.903 & 0.048 \\
\hline \multirow[t]{4}{*}{ Penthiopyrad } & $\begin{array}{l}0.036(0.018-0.056) \\
0.1\end{array}$ & $\begin{array}{l}0(0-0) \\
0\end{array}$ & $\begin{array}{l}0(0-0.009) \\
0.036\end{array}$ & $\begin{array}{l}0(0-0) \\
0.011\end{array}$ & 0.001 & $<0.001$ \\
\hline & Post hoc test & \multicolumn{2}{|c|}{ Isarco valley: Adige valley } & & $<0.001$ & \\
\hline & & \multicolumn{2}{|l|}{ Isarco valley: Low Adige } & & 0.025 & \\
\hline & & \multicolumn{2}{|l|}{ Isarco valley: Venosta } & & $<0.001$ & \\
\hline
\end{tabular}

Contaminated playgrounds $(N=32)$ are stratified for the four investigated valleys. Post hoc test for $n=32$

a Kruskal-Wallis test with post hoc pairwise test for multiple comparisons of mean rank sums (Nemenyi-tests)

b Including all sampling sites $(n=71)$

$\left(R^{2}=0.8\right.$, Table 5): global irradiance $\left(\mathrm{W} \mathrm{m} \mathrm{m}^{-2}\right)$, amount of apple orchards (\%), mean wind blowing to the agricultural field and away from the playground (h), rainfall $(\mathrm{mm})$, distance from agricultural field $(\mathrm{m})$, first grade interaction of distance from agricultural field $(\mathrm{m})$ with maximum wind speed $\left(\mathrm{m} \mathrm{s}^{-1}\right)$, hours of wind when spraying is not recommended (speed $<3 \mathrm{~m} \mathrm{~s}^{-1}$ ), and the first grade interaction between the hours when spraying is allowed and wind speed $\left(\mathrm{m} \mathrm{s}^{-1}\right)$ (Table 5 and Fig. 2).

Due to the higher degree of explained variance in pesticide concentrations, environmental data of the 5-day period before sampling were taken into consideration rather than those of the longer period. Air temperature showed a strong bivariate correlation with the area of apple and wine orchards within a radius of $500 \mathrm{~m}$, respectively, representing the different weather and microclimate structures of the four valleys, and was thus excluded from the model. Playgrounds' altitude (m a.s.l.) showed high VIFs, correlated with meteorological data (wind, temperature, irradiance) and was excluded as well. According to the final model, increasing pesticide contamination of playgrounds was best explained by increasing proportions of apple orchards around the playgrounds and increasing rainfall.
Pesticide contamination was also positively associated with interactions between distance and maximum wind speed, as well as interactions between hours allowed for spraying and prevailing wind speed. In contrast, significant negative associations for pesticide contamination was found for irradiance, wind blowing away from the playgrounds, hours when spraying is not recommended, and the distance to agricultural fields (Table 5 and Fig. 3).

\section{Discussion}

Endocrine-active pesticides on playgrounds-possible impact on human health

This study is among the first demonstrating pesticide contamination in public playgrounds near intensively managed agricultural areas. Pesticide residues were found in $45 \%$ of the sampled playgrounds, while $24 \%$ of the playgrounds were contaminated with more than one compound; in total 12 different pesticides were detected. Overall, 11 of the 12 identified pesticides are endocrineactive substances, which were present in $24 \%$ of the investigated playgrounds [26]. Three detected pesticides, cypermethrin, oxadiazon and tetraconazole, are endocrine disruptors (Category 1, ED confirmed in animal studies, WHO/IPCS definition); chlorpyrifos-methyl, 
Table 5 The results of the multivariate weighted linear regression analysis displaying the associations between pesticide concentration and environmental factors

\begin{tabular}{|c|c|c|c|}
\hline OLS linear regression model & $\begin{array}{l}\text { Estimates } \\
(\beta \text {-weight })\end{array}$ & $95 \% \mathrm{Cl}[\mathrm{LL}, \mathrm{UL}]$ & $p$-value \\
\hline Intercept & 1.0 & 0.35 to 1.65 & 0.004 \\
\hline $\begin{array}{l}\text { Distance from agricultural field } \\
(\mathrm{m})\end{array}$ & -1.07 & -1.99 to -0.16 & 0.023 \\
\hline Apple orchard (\%) & 0.45 & 0.07 to 0.83 & 0.022 \\
\hline Global irradiance $\left(\mathrm{W} \mathrm{m}^{-2}\right)$ & -1.22 & -1.82 to -0.63 & $<0.001$ \\
\hline $\begin{array}{l}\text { Time when spraying not allowed } \\
(\mathrm{h})^{\mathrm{a}}\end{array}$ & -1.03 & -1.45 to -0.61 & $<0.001$ \\
\hline $\begin{array}{l}\text { Wind blowing away from play- } \\
\text { ground }(h)\end{array}$ & -1.23 & -1.92 to -0.55 & 0.001 \\
\hline Rainfall (mm) & 0.62 & 0.20 to 1.04 & 0.006 \\
\hline $\begin{array}{l}\text { Distance: high wind speed } \\
\left(\mathrm{m} \mathrm{s}^{-1}\right)\end{array}$ & 1.25 & 0.48 to 2.02 & 0.003 \\
\hline $\begin{array}{l}\text { Time when spraying allowed (h): } \\
\text { Wind speed }\left(\mathrm{m} \mathrm{s}^{-1}\right)^{\mathrm{a}}\end{array}$ & 0.81 & 0.31 to 1.31 & 0.003 \\
\hline
\end{tabular}

Basic model information and model fit statistics

\begin{tabular}{|c|c|c|c|c|c|c|c|}
\hline$N$ & $F_{(8,21)}$ & AIC & BIC & $R^{2}$ and $C l$ & adj. $R^{2}$ & Residual standard error & $\begin{array}{l}\text { Homoscedasticity (Breusch- } \\
\text { Pagan) }\end{array}$ \\
\hline 69 & 14.75 & 84.53 & 98.54 & $\begin{array}{l}0.85 \\
0.59-0.87\end{array}$ & 0.79 & 1.12 & $\begin{array}{l}\text { Assumption not violated } \\
\qquad(P=0.066)\end{array}$ \\
\hline
\end{tabular}

The model contains first-order interactions showing estimates ( $\beta$ weights) and $95 \%$ confidence intervals ( $95 \% \mathrm{Cl}$ ) for all predictors, as well as indicators for model quality $\left(R^{2}, \mathrm{AIC}, \mathrm{BIC}\right)$. All included predictors are significantly associated with pesticide concentration on playgrounds ( $\mathrm{mg} \mathrm{kg} \mathrm{k}^{-1}=\mathrm{ppm}$. The dependent outcome variable 'pesticide concentration ( $\mathrm{mg} \mathrm{kg}^{-1}$ )' on playgrounds was weighted by the number of detected pesticides per playground. $\mathrm{LL}$, lower level; $\mathrm{UL}$, upper level A significant $b$-weight indicates that semi-partial correlation is also significant. $L L$ and $U L$ indicate the lower and upper limits of a confidence interval, respectively

a Pesticide spraying is allowed during wind speeds $<3 \mathrm{~m} \mathrm{~s}^{-1}$

fluazinam, penthiopyrad and methoxyfenozide are suspected endocrine disruptors (Category 2, ED in-vitro confirmed) and difenoconazole, imidacloprid, penconazole and phosmet are endocrine-active substances or potential ED belonging to Category 3 [27, 54, 62].

The insecticide phosmet and the broad-scale fungicide fluazinam were the most abundant pesticides showing higher concentrations, particularly in the Venosta valley. Both pesticides are used to treat apple trees and grapevines. This unevenly distributed occurrence suggests an application of higher concentrations due to either different climatic conditions in that particular valley (Additional file 1: Fig. S1), and/or higher pest infestations during or prior to the sampling period.

Phosmet and fluazinam are endocrine-active substances and are reported on the endocrine disruptive compounds (EDC) criteria with level 3 and 2, respectively $[4,26]$. According to the EDC screening program, fluazinam is an oestrogen receptor (ER)-antagonist and shows ER bioactivity [71].

Difenoconazole and penthiopyrad, both fungicides, were only found in the Isarco valley, a colder and more rain-laden region, suggesting that the orchards were affected by fruit-specific fungi before and during sampling period.

Dodine was the only detected pesticide without a link to possible ED activity. However, dodine was related to toxicity to humans, including carcinogenicity, reproductive and developmental toxicity, neurotoxicity, and acute toxicity $[8,12,62]$.

Not much is known about effects by exposure to multiple substances. It is known, however, that the exposure to an increasing number of EDs in the environment is associated with an increasing incidence of hormonedependent cancers like breast, prostate and thyroid and a decreasing sperm quality in the European and US population $[24,68,69,86]$. It is important to emphasize that this study only analysed active components and that no consideration was given to metabolites and adjuvants, although these have also been classified as highly toxic $[33,50]$. Children may get in contact through grass and soil at the playground and might additionally be exposed to pesticides through inhalation. Furthermore, places like playgrounds are visited especially by vulnerable groups (i.e., children and pregnant women) and the exposure time may be longer than in other areas $[25,81]$. So far, however, no pesticide threshold levels for air, non-target 


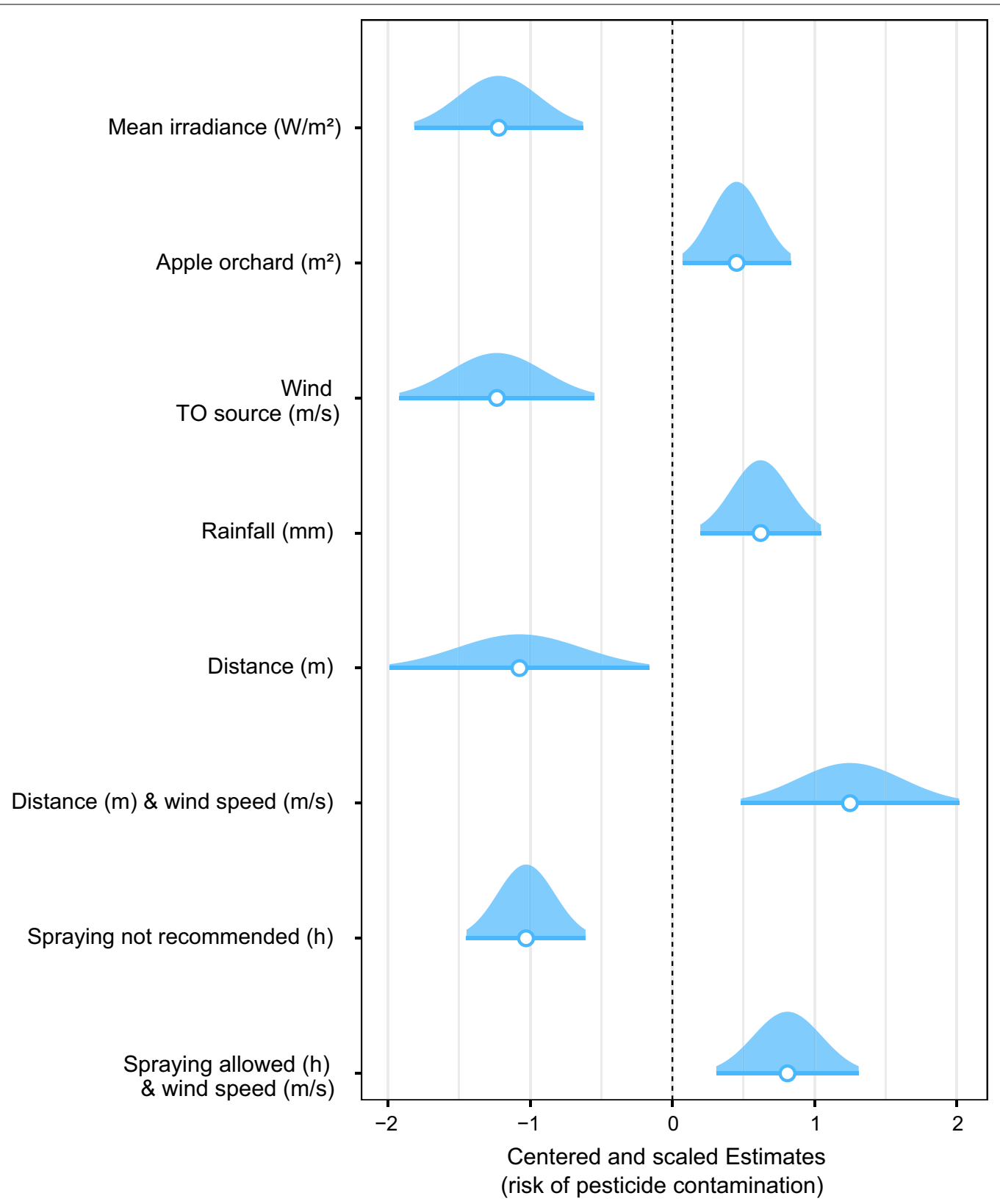

Fig. 2 Forest plot for results of the weighted multiple regression analysis. The left side of the plot (0-2 estimates) shows all factors reducing the risk (possibility) for pesticide contamination while the factors with positive estimates (right side) present risk factors for higher contamination levels. Distributions of each factors are plotted too

vegetation or soil are defined. Although detected concentrations are relatively low, measured concentrations were close to permitted residual levels of fruits and vegetables: the maximum residual levels of fluazinam range from $0.01 \mathrm{ppm}$ for citrus fruit to $0.3 \mathrm{ppm}$ for apples (European [29]); median levels of fluazinam on playgrounds were $0.01 \mathrm{ppm}$ and maximum levels were $0.26 \mathrm{ppm}$. One playground in the Venosta and one playground in the Isarco valley had higher concentrations than the residual levels for citrus fruits and similar to the residual levels of apples. Both playgrounds were near to agricultural sites. Also, the measured levels of tetraconazole and oxadiazon were very close to maximum residual levels (European [29]). Although it is unlikely that substantial amounts of contaminated grass are eaten by children, they may nevertheless get in contact with these substances. The 

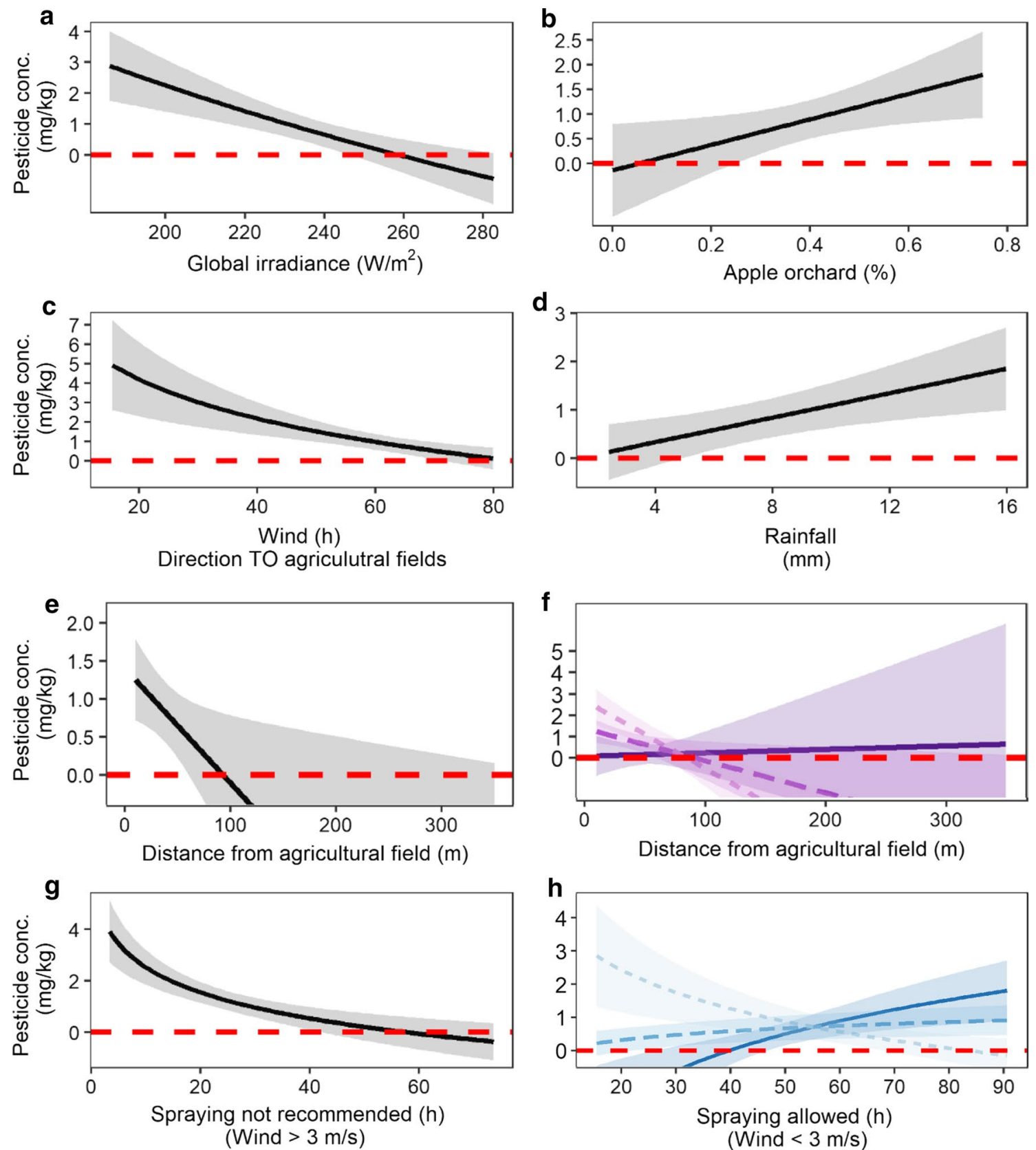

(Wind $>3 \mathrm{~m} / \mathrm{s})$

Wind $(\mathrm{m} / \mathrm{s})$

Wind $\max (\mathrm{m} / \mathrm{s})$

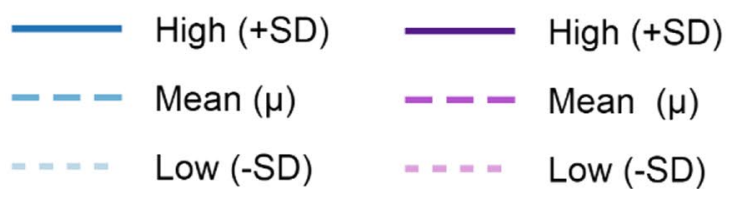

Fig. 3 Effect plots (a-e and $\mathbf{g}$ ) and interaction plots ( $\mathbf{f}$ and $\mathbf{h}$ ) for all factors of the weighted multivariate regression model predicting pesticide concentrations ( $\mathrm{mg} \mathrm{kg}^{-1}=\mathrm{ppm}$ ) with $95 \% \mathrm{Cl}$. Left side: factors reducing pesticide contamination, right side: factors increasing pesticide contamination. For the model the dependent variable pesticide concentration and the factors Wind (h) direction TO agricultural fields, "Spraying not

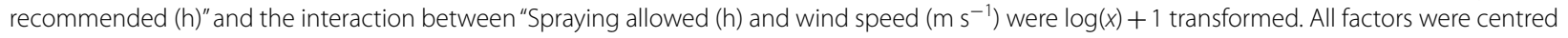
and scaled. Axes show original scales 
concentrations reported here only allow for an overall statement that this concentrations might lead to relevant exposure scenarios for children. An important recommendation for future assessments would be to identify possible uptake pathways by direct ingestion and by uptake via the skin to find meaningful transfer factors, which allow for comparisons with relevant endocrine disruptor concentrations.

The obtained residual levels of these playgrounds are also likely for private gardens or grazing land next to agricultural sites. Therefore, also herbivore productive livestock feeding up to $100 \mathrm{~kg}$ and more grass per day are likely exposed to pesticide residues [32]. Concerning pesticide types and concentrations, our findings are comparable with those published in a recent Austrian study based on field margins [49], showing that insects like butterflies are endangered through pesticide exposure. Furthermore, earlier studies already showed that pesticide application decreased plant biodiversity [67]. Such pollutants disturb the natural processes and can even disrupt ecosystem functioning [15, 23]. However, future studies are needed to shed light on the effects of these substances in the environment.

More important than comparing the findings with permitted residue levels in food is the fact that the majority of pesticides on playgrounds are considered endocrine active because these substances can act like natural hormones in concentrations several orders of magnitude below common residue thresholds [76]. Additional mixtures of EDs at low dose produce significant combination effects also at levels well below the so-called no observed adverse effect level (NOAEL) [47] and it is still discussed whether the long-term exposure of low-dose ED mixtures is associated with chronic diseases [9, 34, 38]. Due to the low-dose effect of EDs, the non-monotonic doseresponse relationship, and the interaction of EDCs with endogenous hormones, it is questionable whether even low doses of EDC can ever be considered as safe $[57,76]$. Thus, a zero tolerance of pesticide residues with ED activity on non-targeted-areas would be comprehensible. So far, the current testing of pesticides with ED activity did not include low-dose and mixture effects, although new testing approaches are described [19]. With this study, we provide additional data on environmental contamination levels pointing to the necessity of future tests.

Pesticide contamination at playgrounds was only once investigated in Europe [66] and at a school yard in the USA [51] so far. Sapcanin et al. [66] found 42 different pesticides with concentrations up to $0.1 \mathrm{mg} \mathrm{kg}-1$ in soil samples, and Miersma et al. [51] reported up to $0.06 \mathrm{mg} \mathrm{kg}^{-1}$ of DDE in soil samples of school yards [51]. However, both studies did not assess pesticide drift from nearby agricultural sites. In addition, the divergence in the detected pesticide types can be attributed to the diverse geographical locations including different pesticide regulations and probably to the type of analysed sample (grass), exhibiting differing degradation processes as compared to soil samples.

Besides pesticides, we also found one preservative agent (2-phenylphenol) and one germicide (benzalkonium chloride). The preservative agent 2-phenylphenol is used for post-harvesting treatment of citrus fruits to protect against microbial damage during storage and distribution [46]. The preservative agent was only detected once in Low Adige near Bolzano at a playground located 100 metres away from an agricultural site. Although this substance is no longer permitted as food additive in Italy [26], it is widely used from households to hospitals, farms and food processing plants and is used for disinfection of seed boxes and food packages and in the production of other fungicides. Furthermore, 2-phenylphenol is found in low concentrations in some household products, such as spray disinfectants and aerosols or deodorants $[16,59]$. Similarly, benzalkonium chloride is a general disinfectant also present in sanitizing agents of all kinds.

Effects of non-occupational, long-term and low-dose pesticide exposure, however, are difficult to establish, as first many toxicological models do not comprehensively consider the numerous variables that can lead to diseases [34] and second it is difficult to account for the total pesticide exposure through all possible exposure routes (e.g., wind, rain, food, work). Most studies on non-occupational pesticide exposure have focussed on pesticide residues in food products and exposure via nutrition [37, $40,72]$, whereas the pesticide exposure via drift is often neglected [77, 87].

\section{Drift of pesticides - an underestimated fact}

This study demonstrated that topographical and meteorological conditions like land use, rainfall, wind speed and its direction influence pesticide drift. Further, precipitation was shown to be positively associated with pesticide concentration. Besides the known direct deposition of pesticides via rainfall [63, 79], local weather conditions (i.e., higher precipitation and wet situations) might in addition motivate farmers to apply higher amounts of pesticides to counteract pest infestations, especially of fungi. As this study found a negative relationship between pesticide concentration and the distance to agricultural areas and a positive association between distance and the interaction with wind, we assume that direct drift via wind might be the dominant vector for pesticides ending up on the playgrounds. Hence, our results strongly suggest that other areas in similar distance to agricultural sites, such as organic fields, private gardens 
or other public spaces, are likely to be contaminated with similar residues and concentration ranges [81].

Our results show that the risk of pesticide contamination on playgrounds is lower at times with elevated wind speeds, when spraying is not allowed (Fig. 3g). In contrast, the combination of long periods of lower wind speeds $\left(0-3 \mathrm{~m} \mathrm{~s}^{-1}\right)$, together with rising wind speed up to $3 \mathrm{~m} \mathrm{~s}^{-1}$, significantly increased the contamination risk, suggesting that the farmers comply with the spray regulations regarding wind. The finding that global irradiance is negatively associated with pesticide contamination refers to pesticide degradation. The fact that the 5-day period prior to sampling had a stronger impact on pesticide concentration than data from the longer period lets suggest that the pesticides detected in this survey might degrade in a sufficient way. However, additional spraying events during the growing season may lead to accumulation of certain pesticides over the vegetation period.

The possibility that the pesticide residues on the playgrounds are partly due to a direct application can be excluded $[39,52]$, as the quantified pesticide concentrations are strongly associated with location (i.e., proportion of apple orchards in the vicinity) and local environmental conditions (i.e., hours of wind conditions when spraying is allowed) and are thus pointing towards a contamination by the surrounding pesticide-treated agricultural areas. Furthermore, most of the detected substances are only allowed for use in agriculture [26, $53,62]$ that theoretically precludes the possibility of contamination by non-commercial use as the application on nearby private land or by community workers.

Besides drift, the aerial transport of volatile compounds is another pesticide pathway to non-target areas. Due to missing information about spraying events and frequencies, however, we could not include volatilisation data of the monitored compounds. In general, existing regulations demand the minimization of volatilization during pesticide spraying and application [28]. There are databases on compound degradation modelling metabolism in soils [35], but data on real compound amounts, as the European Commission regulation 283/2013 claims for, are still lacking. Our study reduces this knowledge gap. To comply this regulation, however, it is still essential that European member states initiate and support more comprehensive field studies.

The predicted impact of each environmental factor shows how pesticide contamination can be reduced with a careful planning, which considers local weather conditions. Our results show that a distance of at least 100 meters is necessary to obtain zero pesticide contamination (Fig. 3e). However, upper confidence intervals indicate that pesticide contamination is also likely within a distance of more than $300 \mathrm{~m}$ and that the additional impact of strong winds can lead to a maximum pesticide concentration up to 5 or more $\mathrm{mg} \mathrm{kg}^{-1}$ (Fig. 3e, f). Therefore, we highly recommend to take into account at least a distance of minimum $100 \mathrm{~m}$ to agricultural sites (Fig. 3e) and also to consider typical and local wind directions when planning and building public places like playgrounds, schools, pre-school and market areas. In addition, higher irradiance and dry weather conditions both reduced pesticide contamination (Fig. 3a, d). That is why we highly recommend to plan and conduct spraying events only during a longer period of sunshine without rainfall and low wind.

\section{Conclusion}

This is a first attempt to assess the susceptibility of public non-agricultural areas for pesticide drift and to examine potential underlying factors. In conclusion, this study demonstrates that pesticides drift from surrounding agricultural fields into playgrounds and that higher fractions of apple orchards in the landscape, rainfall as well as windy conditions after wind-still periods increase pesticide contamination of these sites. Contamination of playgrounds is especially critical because children are very sensitive to EDs and the effects of EDs are considered to have very low thresholds or even do not exist for individual EDs.

It is very likely that other non-crop areas (e.g. private gardens) are affected in a similar way, but this remains to be investigated. Considering the results of this study, combined with the known effects of EDs and drift, extensive and independent surveys using passive samplers to monitor pesticide drift are necessary to ensure sustainability of agricultural and natural ecosystems as well as of public health.

\section{Highlights}

- The screening of 71 public playgrounds in South Tyrol (Italy) showed that $45 \%$ of the playgrounds were contaminated with one or more pesticides.

- In total 12 different pesticides were found with Fluazinam (fungicide) and phosmet (insecticide) as the most abundant ones.

- $92 \%$ of the detected pesticides were endocrine active and $71 \%$ are classified as endocrine disruptors.

- Pesticide contamination was associated with distance and aerial proportion of apple orchards in the surroundings, rainfall, global irradiance, wind speed and direction. 


\section{Additional files}

Additional file 1: Fig S1. Sampled playgrounds at Venosta and the Isarco valley located at significantly higher altitudes above sea level than playgrounds in the Adige valley or the Low Adige ( $F$-value $=63.5, p<0.001$, d.f. $=3$ ).

Additional file 2: Fig S2. The mean distance to agricultural fields $(\mathrm{m})$ from sampled playgrounds does not differ between the four sampling regions $(F$-value $=0.37, p<0.775, d . f .=3)$.

Additional file 3: Fig S3. Percentage of land use [\%] in the four regions of the investigation area in South Tyrol.

\section{Abbreviations}

EDs: endocrine disruptors; a.s.l.: above sea level; AIC: Akaike Information Criterion; BIC: Bayesian Information Criterion; VIF: variance inflation factor; EDC: endocrine-disruptive compounds; ER: oestrogen receptor; ADI: acceptable daily intake; NOAEL: no observed adverse effect level.

\section{Authors' contributions}

CL planed and conducted data analyses, organized explanatory data from local authorities, calculated and selected statistical models, generated tables and graphs and did the literature search. GHN contributed to the statistical analysis and generated the study site graph. MN provided substantial constructive critics and expert knowledge. RN contributed to the descriptive statistics. VT provided substantial knowledge and literature for EDCs. TB helped with the literature search. TW was involved in study organization and study design. AR was involved in field-work organization and study design. JGZ provided the literature and substantial expert knowledge. PC was involved in study organization and design. $\mathrm{KH}$ designed and organized the study and the field work, did preliminary data analysis, helped to organize explanatory data and provided literature. All authors wrote and edited the manuscript. All authors read and approved the final manuscript.

\section{Author details}

${ }^{1}$ Institute of Pharmacy/Pharmacognosy, Leopold-Franzens University Innsbruck, Innsbruck, Austria. ${ }^{2}$ Department of Ecology, University of Innsbruck, Innsbruck, Austria. ${ }^{3}$ Institute of Microbiology, University of Innsbruck, Innsbruck, Austria. ${ }^{4}$ Department of Neurology, Medical University of Innsbruck, Innsbruck, Austria. ${ }^{5}$ Leibniz Institute for Prevention Research and Epidemiology-BIPS, Bremen, Germany. ${ }^{6}$ Council for Research and Experimentation in Agriculture, Research Centre for Animal Production and Aquaculture (CREAZA), Lodi, Italy. ${ }^{7}$ Umbrella Organisation for Nature Conservation and Environmental Protection in South Tyrol-Dachverband für Natur- und Umweltschutz in Südtirol-Federazione Protezionisti Sudtirolesi-Lia Provinziela per Defënder la Natura Südtirol, Bolzano, Italy. ${ }^{8}$ Institute of Zoology, University of Natural Resources and Life Sciences Vienna (BOKU), Vienna, Austria. ${ }^{9}$ Pesticide Action Network Germany, Hamburg, Germany. ${ }^{10}$ Pesticide Action Network Europe, Brussels, Belgium.

\section{Acknowledgements}

We like to thank Angeliki Lyssimachou and Daniela Schuster for their help regarding questions about EDs and Matthias Rainer regarding questions about UNI-EN 15662:2009. We also gratefully acknowledge the University of Innsbruck for co-funding the publication fees for open access.

\section{Competing interests}

The authors declare that they have no competing interests.

\section{Availability of data and materials}

The datasets generated and analysed during the study are not publicly available but are available from the corresponding author on reasonable request.

\section{Consent for publication}

Not applicable.

\section{Ethics approval and consent to participate}

Not applicable.

\section{Funding}

This work was financially supported by the Dachverband für Natur- und Umweltschutz in Südtirol.

\section{Publisher's Note}

Springer Nature remains neutral with regard to jurisdictional claims in published maps and institutional affiliations.

Received: 8 March 2019 Accepted: 20 April 2019

Published online: 08 May 2019

\section{References}

1. Agriculture Victoria, 2017. A guide to estimating wind speeds | For spraying agricultural chemicals [WWW Document]. Victoria State Gov. http:// agriculture.vic.gov.au/agriculture/farm-management/chemical-use/agric ultural-chemical-use/spraying-spray-drift-and-off-target-damage/repor ting-spray-drift-of-agricultural-chemicals/a-guide-to-estimating-windspeeds. Accessed 31 Jan 2019

2. Alavanja MCR, Ross MK, Bonner MR (2013) Increased cancer burden among pesticide applicators and others due to pesticide exposure. CA Cancer J Clin 63:120-142. https://doi.org/10.3322/caac.21170

3. Anastassiades M, Lehotay SJ, Štajnbaher D, Schenck FJ (2003) Fast and easy multiresidue method employing acetonitrile extraction/partitioning and "dispersive solid-phase extraction" for the determination of pesticide residues in produce. J AOAC Int 86:412-431. https://doi.org/10.1371/ journal.pone.0029268

4. Andersson N, Arena M, Auteri D, Barmaz S, Grignard E, Kienzler A, Lepper P, Lostia AM, Munn S, Parra Morte JM, Pellizzato F, Tarazona J, Terron A, Van der Linden S (2018) Guidance for the identification of endocrine disruptors in the context of Regulations (EU) No 528/2012 and (EC) No 1107/2009. EFSA J. 16. https://doi.org/10.2903/j.efsa.2018.5311

5. Atwood D, Paisley-Jones C (2017) Pesticides industry sales and usage 2008-2012 market estimates. US Environmental Protection Agency, Washington, DC, p 20460

6. Autonomous Province Bozen/Bolzano - Soouth Tyrol, A. 9 I., (2017) Geobrowser 3 [WWW Document]. http://gis2.provinz.bz.it/geobrowser. Accessed 30 Jan 2019

7. Bailey HD, Fritschi L, Infante-Rivard C, Glass DC, Miligi L, Dockerty JD, Lightfoot T, Clavel J, Roman E, Spector LG, Kaatsch P, Metayer C, Magnani C, Milne E, Polychronopoulou S, Simpson J, Rudant J, Sidi V, Rondelli R Orsi L, Kang AY, Petridou E, Schüz J (2014) Parental occupational pesticide exposure and the risk of childhood leukemia in the offspring: Findings from the childhood leukemia international consortium. Int J Cancer 135:2157-2172. https://doi.org/10.1002/ijc.28854

8. Band PR, Abanto Z, Bert J, Lang B, Fang R, Gallagher RP, Le ND (2011) Prostate cancer risk and exposure to pesticides in British Columbia Farmers. Prostate 71:168-183. https://doi.org/10.1002/pros.21232

9. Barouki R, Melén E, Herceg Z, Beckers J, Chen J, Karagas M, Puga A, Xia Y, Chadwick L, Yan W, Audouze K, Slama R, Heindel J, Grandjean P, Kawamoto T, Nohara K (2018) Epigenetics as a mechanism linking developmental exposures to long-term toxicity. Environ Int 114:77-86. https:// doi.org/10.1016/j.envint.2018.02.014

10. Bassil KL, Vakil C, Sanborn M, Cole DC, Kaur JS, Kerr KJ (2007) Cancer health effects of pesticides. Can Fam Physician 53:1704-1711

11. Bioprogramm, 2011. Bioprogramm - Biotecnologie avanzate e tecniche ambientali [WWW Document]. http://www.bioprogramm.it/index.jsp. Accessed 30 Jan 2019

12. Börzsönyi M, Pintér $A$, Surján A, Török G (1978) Carcinogenic effect of a quanidine pesticide administered with sodium nitrite on adult mice and on the offspring after prenatal exposure. Cancer Lett 5:107-113

13. Bottoni P, Grenni P, Lucentini L, Caracciolo AB (2013) Terbuthylazine and other triazines in Italian water resources. Microchem J 107:136-142. https ://doi.org/10.1016/J.MICROC.2012.06.011

14. Brouwer M, Kromhout H, Vermeulen R, Duyzer J, Kramer H, Hazeu G, de Snoo G, Huss A (2017) Assessment of residential environmental exposure to pesticides from agricultural fields in the Netherlands. J Expo Sci Environ Epidemiol. https://doi.org/10.1038/jes.2017.3 
15. Burrows LA, Edwards CA (2002) The use of integrated soil microcosms to predict effects of pesticides on soil ecosystems. Eur I Soil Biol 38:245-249. https://doi.org/10.1016/S1164-5563(02)01153-6

16. Coelhan M, Bromig KH, Glas K, Roberts AL (2006) Determination and levels of the biocide ortho-phenylphenol in canned beers from different countries. J Agric Food Chem 54:5731-5735. https://doi.org/10.1021/ jf060743p

17. Costa EMF, Spritzer PM, Hohl A, Bachega TASS, Costa EMF, Spritzer PM, Hohl A, Bachega TASS (2014) Effects of endocrine disruptors in the development of the female reproductive tract. Arq Bras Endocrinol Metabol 58:153-161. https://doi.org/10.1590/0004-2730000003031

18. Damalas CA, Eleftherohorinos IG (2011) Pesticide exposure, safety issues, and risk assessment indicators. Int J Environ Res Public Health 8:14021419. https://doi.org/10.3390/ijerph8051402

19. Day P, Green RM, Gross M, Weltje L, Wheeler JR (2018) Endocrine disruption: current approaches for regulatory testing and assessment of plant protection products are fit for purpose. Toxicol Lett 296:10-22. https:// doi.org/10.1016/J.TOXLET.2018.07.011

20. Decherf S, Demeneix BA (2011) The obesogen hypothesis: a shift of focus from the periphery to the hypothalamus. J Toxicol Environ Health Part B 14:423-448. https://doi.org/10.1080/10937404.2011.578561

21. Department for Environmental Food and Rural Affairs University of Hertfordshire, 2011. Pesticides—best practice guides_avoiding drift [WWW Document]. Univ. Hertfordsh. http://adlib.everysite.co.uk/adlib/defra/ content.aspx?id=000IL3890W.16NTBWTW6M4U3. Accessed 31 Jan 2019

22. Dhouib I, Jallouli M, Annabi A, Marzouki S, Gharbi N, Elfazaa S, Lasram MM (2016) From immunotoxicity to carcinogenicity: the effects of carbamate pesticides on the immune system. Environ Sci Pollut Res 23:9448-9458. https://doi.org/10.1007/s11356-016-6418-6

23. Edwards CA (2002) Assessing the effects of environmental pollutants on soil organisms, communities, processes and ecosystems. Eur J Soil Biol 38:225-231. https://doi.org/10.1016/S1164-5563(02)01150-0

24. El-Zaemey S, Heyworth J, Fritschi L (2013) Noticing pesticide spray drift from agricultural pesticide application areas and breast cancer: a case-control study. Aust N Z J Public Health 37:547-555. https://doi. org/10.1111/1753-6405.12111

25. Elgethun K, Fenske RA, Yost MG, Palcisko GJ (2003) Time-location analysis for exposure assessment studies of children using a novel global positioning system instrument. Health Perspect Environ. https://doi. org/10.1289/ehp.5350

26. European Commission (2017) EU Pesticides database-European Commission [WWW Document]. http://ec.europa.eu/food/plant/pesticides /eu-pesticides-database/public/?event=activesubstance.detail\&langu age $=E N \&$ selectedID $=891$. Accessed $22 \mathrm{Jul} 2018$

27. European Commission (2016) Defining criteria for identifying endocrine disruptors in the context of the implementation of the plant protection products regulation and biocidal products regulation. European Commission, Brussels

28. European Commission (2013) Commission Regulation (EU) No 283/2013. Brussels

29. European Commission (2011) Commission regulation (EU) No 1178/2011. Off. J. Eur. Union

30. European Commission Statistical Office-Eurostat (2017) Agricultural production-crops [WWW Document]. http://ec.europa.eu/eurostat/stati stics-explained/index.php/Agricultural_production_-_crops. Accessed 20 Aug 2004

31. European Commission Statistical Office-Eurostat (2012) Agri-environmental indicator-consumption of pesticides [WWW Document]. http://epp. eurostat.ec.europa.eu/statistics_explained/index.php/Agri-environmen tal_indicator_-_consumption_of_pesticides. Accessed 20 Aug 2019

32. Ferré DM, Quero AAM, Hernández AF, Hynes V, Tornello MJ, Lüders C, Gorla NBM (2018) Potential risks of dietary exposure to chlorpyrifos and cypermethrin from their use in fruit/vegetable crops and beef cattle productions. Environ Monit Assess 190:292. https://doi.org/10.1007/s1066 $1-018-6647-x$

33. Foy CL, Pritchard DW (1996) Pesticide formulation and adjuvant technology. Taylor \& Francis, CRC Press, Boca Raton

34. Gangemi S, Miozzi E, Teodoro M, Briguglio G, De Luca A, Alibrando C, Polito I, Libra M (2016) Occupational exposure to pesticides as a possible risk factor for the development of chronic diseases in humans. Mol Med Rep 14:4475-4488. https://doi.org/10.3892/mmr.2016.5817
35. Gao J, Ellis LBM, Wackett LP (2010) The University of Minnesota Biocatalysis/Biodegradation Database: improving public access. Nucleic Acids Res 38:D488-D491. https://doi.org/10.1093/nar/gkp771

36. Geier, G., Peterlin, D., Tartarotti, P., Rastner, L., 2018. Historische Daten | Wetter Südtirol | Autonome Provinz Bozen - Südtirol [WWW Document]. http://wetter.provinz.bz.t//historische-daten.asp. Accessed 29 Oct 2018

37. Gerage JM, Meira APG, da Silva MV (2017) Food and nutrition security: pesticide residues in food. Nutrire 42:3. https://doi.org/10.1186/s4111 0-016-0028-4

38. Hanson MA, Gluckman PD (2008) Developmental origins of health and disease: new insights. Basic Clin Pharmacol Toxicol 102:90-93. https://doi. org/10.1111/j.1742-7843.2007.00186.x

39. Hedblom M, Lindberg F, Vogel E, Wissman J, Ahrné K (2017) Estimating urban lawn cover in space and time: case studies in three Swedish cities. Urban Ecosyst. https://doi.org/10.1007/s11252-017-0658-1

40. Holme F, Thompson B, Holte S, Vigoren EM, Espinoza N, Ulrich A, Griffith W, Faustman EM (2016) The role of diet in children's exposure to organophosphate pesticides. Environ Res 147:133-140. https://doi.org/10.1016/j. envres.2016.02.003

41. Hvězdová M, Kosubová P, Košíková M, Scherr KE, Šimek Z, Brodský L, Šudoma M, Škulcová L, Sáňka M, Svobodová M, Krkošková L, Vašíčková J, Neuwirthová N, Bielská L, Hofman J (2018) Currently and recently used pesticides in Central European arable soils. Sci Total Environ 613-614:361-370. https://doi.org/10.1016/J.SCITOTENV.2017.09.049

42. Istat (2018) Tavola 07 : Principi attivi contenuti nei prodotti fitosanitari per ettaro di superficie trattabile (in chilogrammi). Dettaglio per regione - Anno 2017 - [WWW Document]. http://agri.istat.it/jsp/dawin ci.jsp?q $=$ pl07a000001000001 2000\&an $=2017 \& \mathrm{ig}=1 \& \mathrm{ct}=428 \& \mathrm{id}=3 \mathrm{~A} \% 7 \mathrm{C}$ 45A\%7C66A\%7C67A. Accessed 30 Jan 2019

43. Jacobsen CS, Hjelmsø MH (2014) Agricultural soils, pesticides and microbial diversity. Curr Opin Biotechnol 27:15-20. https://doi.org/10.1016/J. COPBIO.2013.09.003

44. Kab S, Moisan F, Elbaz A (2017) Farming and incidence of motor neuron disease: French nationwide study. Eur J Neurol 24:1191-1195. https://doi. org/10.1111/ene.13353

45. Kachuri L, Harris MA, MacLeod JS, Tjepkema M, Peters PA, Demers PA (2017) Cancer risks in a population-based study of 70,570 agricultural workers: results from the Canadian census health and Environment cohort (CanCHEC). BMC Cancer 17:343. https://doi.org/10.1186/s1288 5-017-3346-X

46. Kim S, Chen J, Cheng T, Gindulyte A, He J, He S, Li Q, Shoemaker BA, Thiessen PA, Yu B, Zaslavsky L, Zhang J, Bolton EE (2018) PubChem 2019 update: improved access to chemical data. Nucleic Acids Res 47:1-8. https://doi.org/10.1093/nar/gky1033

47. Kortenkamp A (2008) Low dose mixture effects of endocrine disrupters: implications for risk assessment and epidemiology. Int J Androl 31:233-240. https://doi.org/10.1111/j.1365-2605.2007.00862.x

48. Laetz CA, Baldwin DH, Collier TK, Hebert V, Stark JD, Scholz NL (2009) The synergistic toxicity of pesticide mixtures: implications for risk assessment and the conservation of endangered Pacific salmon. Environ Health Perspect 117:348-353. https://doi.org/10.1289/ehp.0800096

49. Linhard D, Unterkircher C, Eder H, Potuschak S (2018) Pestizidtest: Ackerrandstreifen. Austria, Vienna

50. Mesnage R, Antoniou MN (2017) Ignoring adjuvant toxicity falsifies the safety profile of commercial pesticides. Front Public Health 5:361. https:// doi.org/10.3389/fpubh.2017.00361

51. Miersma NA, Pepper CB, Anderson TA (2003) Organochlorine pesticides in elementary school yards along the Texas-Mexico border. Environ Pollut 126:65-71. https://doi.org/10.1016/S0269-7491(03)00126-X

52. Milesi C, Running SW, Elvidge CD, Dietz JB, Tuttle BT, Nemani RR (2005) Mapping and modeling the biogeochemical cycling of turf grasses in the United States. Manage Environ. https://doi.org/10.1007/s0026 7-004-0316-2

53. Ministero della Salute (2019) Prodotti fitosanitari - Risultato della ricerca della banca dati dei prodotti fitosanitari [WWW Document]. http:// www.fitosanitari.salute.gov.it/fitosanitariwsWeb_new/FitosanitariServlet. Accessed 31 Jan 2019

54. MnifW, Hassine AlH, Bouaziz A, Bartegi A, Thomas O, Roig B (2011) Effect of endocrine disruptor pesticides: a review. J Environ Res Public Health, Int. https://doi.org/10.3390/ijerph8062265 
55. Muir K, Rattanamongkolgul S, Smallman-Raynor M, Thomas M, Downer S, Jenkinson C (2004) Breast cancer incidence and its possible spatial association with pesticide application in two counties of England. Public Health 118:513-520. https://doi.org/10.1016/J.PUHE.2003.12.019

56. Mulleners WM, Brouwer $M$, van Laar T, Vermeulen $\mathrm{RCH}$, Kromhout $\mathrm{H}$, Huss A, Sas AMG, van der Mark M, de Snoo GR, Nijssen PCG (2017) Environmental exposure to pesticides and the risk of Parkinson's disease in the Netherlands. Int Environ. https://doi.org/10.1016/j.envint.2017.07.001

57. Munn S, Goumenou M (2013) Institute for Health and Consumer Protection. Thresholds for endocrine disrupters and related uncertainties : report of the Endocrine Disrupters Expert Advisory Group. Publications Office

58. Münze R, Hannemann C, Orlinskiy P, Gunold R, Paschke A, Foit K, Becker J, Kaske O, Paulsson E, Peterson M, Jernstedt H, Kreuger J, Schüürmann G, Liess M (2017) Pesticides from wastewater treatment plant effluents affect invertebrate communities. Sci Total Environ 599-600:387-399. https://doi.org/10.1016/j.scitotenv.2017.03.008

59. National Institute of Health (2015) Haz-Map [WWW Document]. U.S. Natl. Libr. Med. https://hazmap.nlm.nih.gov/category-details?id=6418\&table =copytblagents. Accessed 31 Jan 2019

60. Nearest!, R.U. (2017) Spielplatz Finder [WWW Document]. https://playg round.nearest.place/de. Accessed 30 Jan 2019

61. Office-Eurostat ECS (2018) Agri-environmental indicator-consumption of pesticides [WWW Document]. http://epp.eurostat.ec.europa.eu/statistics _explained/inde. Accessed 20 Aug 2004

62. Pesticide Action Network (2016) PAN pesticide database. Oakland, CA

63. Plimmer JR (1990) Pesticide loss to the atmosphere. Am J Ind Med 18:461-466. https://doi.org/10.1002/ajim.4700180418

64. PN-EN 15662, 2008 (2008) Foods of plant origin-determination of pesticide residues using GC-MS and/or LC-MS(/MS) following acetonitrile extraction/partitioning and cleanup by dispersive SPE-QuEChERS-method

65. R Core Team (2018) R: a language and environment for statistical computing. R Found Stat Comput. https://doi.org/10.1007/978-3-540-74686-7

66. Sapcanin A, Cakal M, Imamovic B, Salihovic M, Pehlic E, Jacimovic Z, Jancan G (2016) Herbicide and pesticide occurrence in the soils of children's playgrounds in Sarajevo, Bosnia and Herzegovina. Environ Monit Assess 188:450. https://doi.org/10.1007/s10661-016-5463-4

67. Schmitz J, Hahn M, Brühl CA (2014) Agrochemicals in field margins-an experimental field study to assess the impacts of pesticides and fertilizers on a natural plant community. Agric Ecosyst Environ 193:60-69. https:// doi.org/10.1016/j.agee.2014.04.025

68. Sekhotha MM, Monyeki KD, Sibuyi ME (2016) Exposure to agrochemicals and cardiovascular disease: a review. Int J Environ Res Public Health. https ://doi.org/10.3390/ijerph13020229

69. Soto AM, Sonnenschein C (2010) Environmental causes of cancer: endocrine disruptors as carcinogens. Nat Rev Endocrinol 6:363-370. https:// doi.org/10.1038/nrendo.2010.87

70. Stenert C, de Mello ÍCMF, Pires MM, Knauth DS, Katayama N, Maltchik $L$ (2018) Responses of macroinvertebrate communities to pesticide application in irrigated rice fields. Environ Monit Assess 190:74. https:// doi.org/10.1007/s10661-017-6425-1

71. US Environmental Protection Agency (2016) Endocrine Disruptor Screening Program (EDSP). Environmental Protection Agency, Washington, DC

72. Valcke M, Bourgault M-H, Rochette L, Normandin L, Samuel O, Belleville D, Blanchet C, Phaneuf D (2017) Human health risk assessment on the consumption of fruits and vegetables containing residual pesticides: a cancer and non-cancer risk/benefit perspective. Environ Int 108:63-74. https://doi.org/10.1016/j.envint.2017.07.023

73. Van Hoesel W, Dorn VM, Tiefenbacher A, Koller R, Bonkowski M, Ratzenböck A, Wiklicky V, König N, Widhalm T, Hagenguth JF, Lagerlöf J, Prah U, Zaller JG (2017) Single and combined effects of pesticide seed dressings and herbicides on earthworms, soil microorganisms, and litter decomposition. Front Plant Sci 8:215. https://doi.org/10.3389/fpls.2017.00215
74. Van Maele-Fabry G, Gamet-Payrastre L, Lison D (2017) Residential exposure to pesticides as risk factor for childhood and young adult brain tumors: a systematic review and meta-analysis. Environ Int 106:69-90. https://doi.org/10.1016/j.envint.2017.05.018

75. Van Maele-Fabry G, Libotte V, Willems J, Lison D (2006) Review and metaanalysis of risk estimates for prostate cancer in pesticide manufacturing workers. Cancer Causes Control 17:353-373. https://doi.org/10.1007/ s10552-005-0443-y

76. Vandenberg LN, Colborn T, Hayes TB, Heindel JJ, Jacobs DR, Lee D-H, Shioda T, Soto AM, vom Saal FS, Welshons WV, Zoeller RT, Myers JP, Myers JP (2012) Hormones and endocrine-disrupting chemicals: low-dose effects and nonmonotonic dose responses. Endocr Rev 33:378-455. https ://doi.org/10.1210/er.2011-1050

77. Vinceti M, Filippini T, Violi F, Rothman KJ, Costanzini S, Malagoli C, Wise LA, Odone A, Signorelli C, lacuzio L, Arcolin E, Mandrioli J, Fini N, Patti F, Lo Fermo S, Pietrini V, Teggi S, Ghermandi G, Scillieri R, Ledda C, Mauceri C, Sciacca S, Fiore M, Ferrante M (2017) Pesticide exposure assessed through agricultural crop proximity and risk of amyotrophic lateral sclerosis. Environ Health A Glob Access Sci Source 16:91. https://doi.org/10.1186/s1294 0-017-0297-2

78. VKE (2017) Verein für Kinderspielplätze und Erholung —VKE [WWW Document]. https://www.vke.it/. Accessed 30 Jan 2019

79. Vogel JR, Majewski MS, Capel PD (2008) pesticides in rain in four agricultural watersheds in the United States. J Environ Qual 37:1101. https://doi. org/10.2134/jeq2007.0079

80. Wahab A, Hod R, Ismail N, Omar N (2016) The effect of pesticide exposure on cardiovascular system: a systematic review. Int J Commun Med Public Health 3:1-10. https://doi.org/10.18203/2394-6040.ijcmph20151542

81. Weppner S, Elgethun K, Lu C, Hebert V, Yost MG, Fenske RA (2006) The Washington aerial spray drift study: Children's exposure to methamidophos in an agricultural community following fixed-wing aircraft applications. J Expo Sci Environ Epidemiol. https://doi.org/10.1038/sj.jea.75004 61

82. Ye M, Beach J, Martin JW, Senthilselvan A (2017) Pesticide exposures and respiratory health in general populations. J Environ Sci 51:361-370. https ://doi.org/10.1016/j.jes.2016.11.012

83. Ye M, Beach J, Martin JW, Senthilselvan A (2015) Associations between dietary factors and urinary concentrations of organophosphate and pyrethroid metabolites in a Canadian general population. Int J Hyg Environ Health 218:616-626. https://doi.org/10.1016/j.ijheh.2015.06.006

84. Zaller JG, Cantelmo C, Santos G Dos, Muther S, Gruber E, Pallua P, Mandl K, Friedrich B, Hofstetter I, Schmuckenschlager B, Faber F (2018) Herbicides in vineyards reduce grapevine root mycorrhization and alter soil microorganisms and the nutrient composition in grapevine roots, leaves, xylem sap and grape juice. Environ Sci Pollut Res 25:23215-23226. https://doi. org/10.1007/s11356-018-2422-3

85. Zaller JG, Heigl F, Ruess L, Grabmaier A (2014) Glyphosate herbicide affects belowground interactions between earthworms and symbiotic mycorrhizal fungi in a model ecosystem. Sci Rep 4:5634. https://doi. org/10.1038/srep05634

86. Zeng F, Lerro C, Lavoué J, Huang H, Siemiatycki J, Zhao N, Ma S, Deziel NC, Friesen MC, Udelsman R, Zhang Y (2017) Occupational exposure to pesticides and other biocides and risk of thyroid cancer. Occup Environ Med 74:502-510

87. Zhang X, Luo Y, Goh KS (2018) Modeling spray drift and runoff-related inputs of pesticides to receiving water. Environ Pollut 234:48-58. https:// doi.org/10.1016/j.envpol.2017.11.032

88. Zuur AF, Ineo EN, Walker NJ, Saveliev AA, Smith GM (2009) Mixed effects models and extensions in ecology with $\mathrm{R}$, Springer. https://doi. org/10.1016/B978-0-12-387667-6.00013-0 